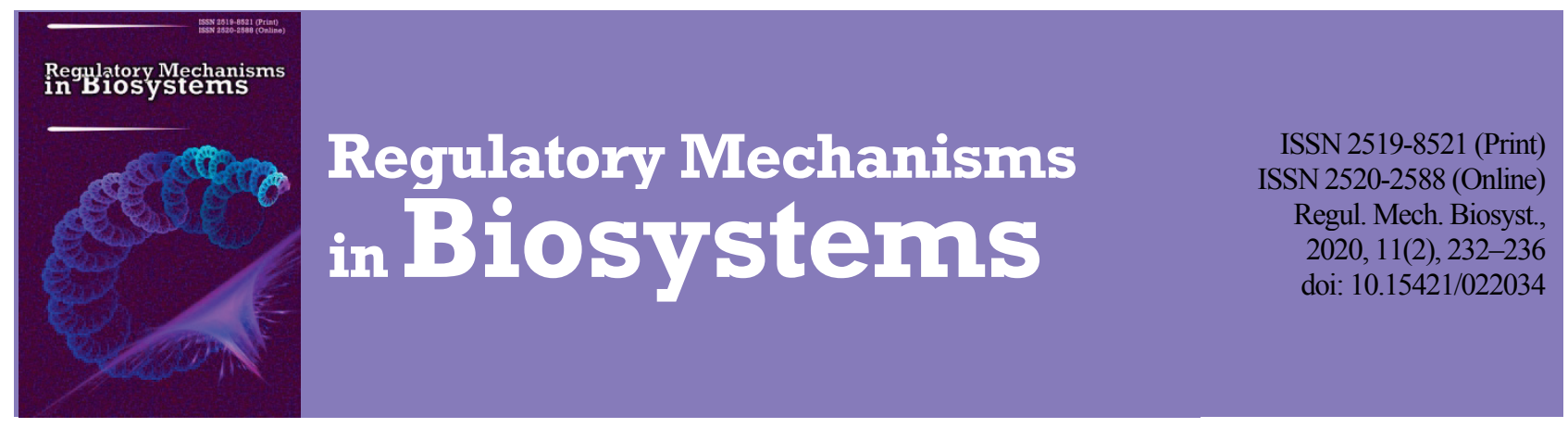

\title{
Phenolic substances as regulators of the intensity of lipid peroxidation processes of the strains Pleurotus eryngii, Fistulina hepatica and Agrocybe cylindracea
}

\author{
O. V. Fedotov \\ Donetsk National Medical University, Kramatorsk, Ukraine
}

Article info

Received 28.03.2020

Received in revised form 21.04.2020

Accepted 22.04.2020

Donetsk National Medical University, Machine-Builders Boulevard, 39,

Kramatorsk, 84331,

Ukraine.

Tel.: +38-068-717-87-87

E-mail: bio.graff@ukr.net

Fedotov, O. V. (2020). Phenolic substances as regulators of the intensity of lipid peroxidation processes of the strains Pleurotus eryngii, Fistulina hepatica and Agrocybe cylindracea. Regulatory Mechanisms in Biosystems, 11(2), 232-236. doi:10.15421/022034

The work is devoted to the calculation, comparison of indicators and the development of a method for regulating the intensity of lipid peroxidation processes (LPP) of strains of basidiomycetes. The purpose of the investigation is to study the effect of phenolic type chemicals and hydrogen peroxide on the lipid peroxidation of certain strains of basidiomycetes under laboratory cultivation. Cultivation of strains of basidiomycetes was carried out by periodic surface method on glucose-peptone medium (GPM) in flasks. The influence of sodium lignosulfonate, tannin, gallic acid and hydrogen peroxide at $0.1 \%$ concentration at 24 and 48 hours of exposure on the intensity of lipid peroxidation processes of the strains Pleurotus eryngii P-er, Fistulina hepatica Fh-08 and Agrocybe cylindracea 960, fungi of the phylum Basidiomycota, orderAgaricales has been investigated. It was established that the used phenolic-type chemical compounds that are part of the lignocellulose complex of wood or are the products of its decomposition to a certain extent affect the lipid peroxidation processes of mycelial cell lipids in the studied cultures of basidium fungi. The individual reaction of LPP of cultures to the applied substance and the time of its exposure are determined. The highest degree of LPP induction was recorded upon addition of tannin - by $161 \%$, after 48 hours of exposure in the mycelium of strain Fh-08; sodium lignosulfonate - by 192\%, after 48 hours in the mycelium of strain P-er; gallic acid - by $182 \%$, after 24 hours, in the mycelium of strain P-er; hydrogen peroxide - by $257 \%$, after 24 hours, in the CR of strain 960. The biotechnological significance of this is the possibility of regulation (induction or repression) of LPP of producer strains. From a biomedical point of view, the possibility of changing the activity of lipid peroxidation processes of mycelium and culture fluid makes it possible to obtain and use more effective functional products of fungal origin.

Keywords: basidium fungi; surface cultivation; lipid peroxidation; regulation;chemical substances.

\section{Introduction}

Reactive oxygen species (ROS) are constantly formed in a living cell as products of normal oxygen metabolism. Various environmental factors, including teratogenic factors, have been identified in this process. The study of this effect is of practical interest, since ROS can play the role of mediators of important intracellular signaling pathways, inducers of the defense system and ion transport systems, trigger programmed cell apoptosis, etc. (Lushchak, 2014; Fedotov \& Bisko, 2018).

However, increased of ROS production leads to the development of oxidative stress and is a serious problem for a living organism. Due to damage to DNA, protein and lipid oxidation, a number of pathogenic processes and rapid aging of individual cells and the whole organism take place. As is well known, the antioxidant system counteracts the ROS and maintains the oxidation-reduction balance of the status of the organism (Bennett et al., 2017; Obrador et al., 2019).

Interest in lipid peroxidation in biological membranes has increased dramatically in recent times. This is evidenced by numerous publications in a wide variety of biological and medical periodicals (Kapich, 2010; Lushchak, 2011; Fedotov \& Bisko, 2018). Just a few years ago, the study of chain reactions of fatty acid peroxidation represented insignificant branches of various branches of science, quite separate from each other. In chemical kinetics, the food industry, in certain issues of pathology, toxicology, radiobiology, biochemistry, biophysics, etc., there have been scattered studies on lipid peroxidation, but any of them taken separately, as it were, is a special case secondary in the scale of each of the listed sciences. But, with the accumulation of facts, it became more and more obvious that the study of the free radical chain oxidation of unsaturated fatty acids in a cell is in itself an important scientific problem, located at the intersection of a number of related disciplines (Lushchak, 2016; Fedotov, 2016). First of all, the problem of LPP in biological membranes, as well as membrane biology in general, lies on the boundary between biochemistry and biophysics. Biochemical methods for extraction of subcellular particles, enzyme control, as well as analysis of peroxidation products, are found to be an important addition to biophysical methods for studying free radical processes, among which in this case the most fruitful was the measurement of chemiluminescence accompanying these processes. The biochemical concept of metabolism, regulated by a number of factors, including enzymes, is supplemented by biophysical categories of free radical reactions in the biomolecular structure of biological membranes (Lushchak, 2016).

The study of LPP has brought together two other sciences: chemical kinetics and pathology (Bennett et al., 2017; Vitaket al., 2017; Obrador et al., 2019). As in the case of other organic compounds, lipid peroxidation is subject to kinetic laws characteristic of chain reactions; they have been studied for objects far from biology, for example, of liquid-phase oxidation of cyclohexane or cumol. But on the other hand, the appearance of peroxide in tissues is one of the important factors of the action of often negative agents (for example, radiation, poisons or vitamin deficiency, etc.) that damage the cells components and membranes. While the investigation of kinetics is carried out on the example of hydrocarbons, and the study of pathology is carried out at the organism level, there is no real connection between these two directions. But, with the transition of research to the level of solutions and suspensions of lipids, biological membranes or organelles, kinetics and pathology become the cause and effect of the same molecular events (Lushchak, 2016). 
Finally, peroxidation processes are of interest to molecular biologists and doctors. The sensational discoveries made in the last twenty years in the field of molecular biology give hope for solving the main problems of theoretical medicine and applying the achievements of biological science to the study of each specific disease. But with this approach, we do not use strategy, but only the weapon of modern biology. Meanwhile, the most important thesis of molecular biology is that the basic processes of cellular activity, such as protein biosynthesis, DNA replication or oxidative phosphorylation, are in principle not only in the cells of the same organism, but also in cells of completely different organisms, for example bacteria and humans. Does this mean that the basis of damage, disease, and death should also be some universal processes of subcellular and cellular pathology common to all cells? Obviously, damage to cell membranes and cell organelles is one of such universal pathological processes. In turn, among the causes of membrane damage, peroxidation of membrane phospholipids is not in last place. However, as we have already noted, the processes of peroxidation undoubtedly play a certain role in the normal functioning of cells (Fedotov, 2016; Bennett et al., 2017).

Most of all, the role, features and regulation of LPP are studied in human and animal cells. Increasingly, there are works on the importance of lipid peroxidation in plant life. As for microorganisms and fungi, they remain poorly researched. At the same time, it is assumed that lipid peroxidation can play an important role in various natural processes carried out by microorganisms and fungi, for example, in the oxidative transformation of various compounds, bioluminescence, etc. (Elisashvili et al., 2018; Metri et al., 2018).

Mushrooms are the most diverse and numerous group of organisms, promising producers of biologically active metabolites for many reasons (Wasser, 2011; Syrchin \& Grodzinskaya, 2015). In particular, the genetic richness of these organisms, the uniqueness of mushroom compounds and their metabolic resemblance to animals, the profitability of the simultaneous production of several products, the unpretentiousness of cultures in nutrient media, the absence of a vast majority of types of sporulation in the culture and the saving of time of the biotechnological process, lack of competition with traditional food sources are noted. The relevance and prospect of attracting strains of fungi to biotechnological production is also evidenced by the growing number of their pharmacological, therapeutic and medical research (Chien et al., 2016; Fedotov, 2016; Wasser, 2017).

A special place among fungi is occupied by a group of xylotrophic basidiomycetes, which play a key role in the circulation of substances and the flow of energy in ecosystems. Recently, large-scale studies have been conducted aimed at studying the mechanisms of lignin breakdown in nature, since it is assumed that knowledge of these mechanisms can open the way for the development of environmentally friendly, energysaving biotechnologies for processing lignocellulosic raw materials (Elisashvili et al., 2018; Han et al., 2018; Metri et al., 2018). It is generally recognized that the destruction of the main components of woodcellulose and lignin - occurs in nature, mainly under the influence of extracellular fungal enzymes (Eriksson et al., 1990; Voloshko, 2011; Vitak et al., 2017). At the same time, it was found that the destruction of wood lignin is the result of free radical oxidation reactions and is accompanied by the formation of ROS. There is experimental evidence of the ability of xylotrophs to form ROS, which can cause destruction of lignin and cellulose. Since free radicals also have the ability to initiate peroxidation of various components of the cell, and primarily lipids, it is important to assess the possibility of occurrence and regulation of LPO processes in cultures of basidiomycetes (Kapich, 2010; Asatiani et al., 2018; Fedotov \& Bisko, 2018).

It is hoped that the knowledge of the regularities of the occurrence of LP processes and their regulation in fungi will enable the development of scientifically based ways to control these processes and optimize biotechnological methods for the production of polyunsaturated compounds of lipid nature, thereby significantly increasing their yield. The results of these researches can also become the scientific foundation for the practical development of new environmentally friendly biotechnologies for biodegradation of chemically resistant polymers, delignification of wood or other types of lignocellulosic raw materials, for ex- ample, in paper production (Fedotov, 2016). Based on the foregoing, the goal of the research is to establish the distribution of strains by level and compare the indicators of the influence of phenolic type chemicals and hydrogen peroxide on the intensity of lipid peroxidation processes in the growth dynamics of basidiomycotic fungi strains by their surface periodic cultivation on a glucose-peptone medium in laboratory conditions.

\section{Material and methods}

On the basis of the results of preliminary screening, biotechnologically valuable 58 strains were selected for 13 species of the phylum Basidiomycota, Polyporales orders: Daedalea quercina Dq-08, Ganoderma lucidum $\mathrm{Gl}-2$, Irpex lacteus $\mathrm{Il}-4 \mathrm{k}$, Fomes fomentarius T-10, Laetiporus sulphureus Ls-08 and Agaricales: Agrocybe cylindracea 167, 218 and 960, Fistulina hepatica Fh-08, Lentinula edodes 523, Flammulina velutipes F-03, F-06, F-073, F-1, F-10, F-102, F-104, F-107, F-112, F-2, F-202, F-204, F-610 and F-vv, Pleurotus citrinopileatus P-ctr, Pleurotus eryngii P-er, Pleurotus ostreatus D-140, Hk-35, P-004, P-01, P-035, P-039, P-081, P-082, P-083, P-087, P-088, P-089, P-105, P-107, P -12k, P-191, P-192, P-203, P-206, P-208, P-209, P-210, P-2175, P447, P-6v, P-kl, P 14 P -4k, P-91, P-94 and P-998, Schizophyllum commune Sc-10 (Velygodska \& Fedotov, 2016; Fedotov \& Bisko, 2018). The systematic position of the strains was established according to modern literature (Kirk et al., 2008). All studied strains are stored in the Collection of Hat Mushroom Cultures at the M. G. Kholodny Institute of Botany National Academy of Sciences of Ukraine (IBK), having the status of the National Heritage of Ukraine (Bisko et al., 2016).

To study the intensity of lipid peroxidation processes of cultures, vegetative mycelium was cultivated using the periodic surface method on glucose-peptone medium (GPM) of the following composition $\left(\mathrm{g} / \mathrm{dm}^{3}\right)$ : glucose - 10.0; peptone - 3.0; $\mathrm{KH}_{2} \mathrm{PO}_{4}-0.6 ; \mathrm{K}_{2} \mathrm{HPO}_{4}-0.4 ; \mathrm{MgSO}_{4} \times$ $7 \mathrm{H}_{2} \mathrm{O}-0.5 ; \mathrm{CaCl}_{2}-0.05 ; \mathrm{ZnSO}_{4} \times 7 \mathrm{H}_{2} \mathrm{O}-0.001$; distilled water - up to $1 \mathrm{dm}^{3}$. Cultivation was carried out in Erlenmeyer flasks with a volume of $250 \mathrm{~cm}^{3}$ containing $50 \mathrm{~cm}^{3}$ of culture medium. The initial value and $\mathrm{pH}$ of the medium after the introduction of chemicals and the cultivation temperature were individual and optimal for each strain depending on the values that were established at the previous stage of research. When studying the effect, on the 10th day of culturing the strains, aqueous solutions of sodium lignosulfonate, tannin, gallic acid and hydrogen peroxide up to $0.1 \%$ were aseptically added to the research flasks, and distilled water in equal volumes was added to the control flasks. At the same time, the $\mathrm{pH}$ of the culture fluid (CF) was controlled, biosynthetic parameters were measured after 24 and 48 hours of exposure to substances (Fedotov \& Bisko, 2018).

The accumulation of absolutely dry biomass (ADB) by cultures, preparation of mycelium and culture fluid, determination of the acidity of culture media, culture fluid and working solutions were fixed using standard methods. The fermentation process of the strains was evaluated by the accumulation and productivity of biomass (Qx) (Fedotov \& Bisko, 2018), the intensity of the LPprocess - by the results of the test with thiobarbituric acid-TBA test for TBA-active products (TBA-AP). In order to determine the level of induced intensity of LPO processes, $1 \times 10^{-3} \mathrm{~mol} / \mathrm{dm}^{3}$ sulfuric acid solution and $1 \times 10^{-2} \mathrm{~mol} / \mathrm{dm}^{3}$ of ascorbic acid were added to the aqueous extract of mycelium or CF (control distilled water) (Fedotov, 2016).

The studies were carried out in triplicate. Data are expressed as mean values \pm error. Statistical analysis was performed using OriginPro 8.5.1 software (Origin-Lab Corportion, USA), and samples were compared using one-way ANOVA. The difference $\mathrm{P}<0.05$ was considered statistically significant compared with the control.

\section{Results}

It was found that the spontaneous intensity of the LPP (LPPs) processes of the mycelium of all strains was significantly higher than this indicator in the culture fluid. The intensity of lipid peroxidation of mycelium increased with the age of the culture, which can be explained by a growing shortage of certain nutrients (primarily carbon-containing) and an increase in the number and concentration of metabolic products 
in the culture fluid. According to the level of unauthorized intensity of the LP processes of a 12-day-old mycelium, the studied strains can be divided into three conditional groups (Fig. 1).

9 strains belong to the first group with a low level of unauthorized intensity of LP processes - below $40 \mathrm{nmol} / \mathrm{g}$ ADB. The recorded values of Ac are probably associated with insignificant indicators of their growth and corresponding biosynthetic processes. The largest group, with moderate Ac values from 40 to $80 \mathrm{nmol} / \mathrm{g} A D B$, includes 32 strains. The third conditional group includes 17 cultures with a high content of TBA-AP - more than $80 \mathrm{nmol} / \mathrm{g}$. These are strains of various systematic affiliation $D$. quercina Dq-08, F. fomentarius T-10, A. cylindracea 960, L. edodes 523, F. hepatica Fh-08, F velutipes F-202, P. citrinopileatus P-citr, P. eryngii P-er, $P$. ostreatus P-035, P-089, P191, P-kl, P-14, P-94, P-447 and P-2175 and S. commune Sc-10. Based on the high intensity of LP processes recorded, these cultures have prospects for the use of chemically stable compounds in the destruction of biotechnologies.
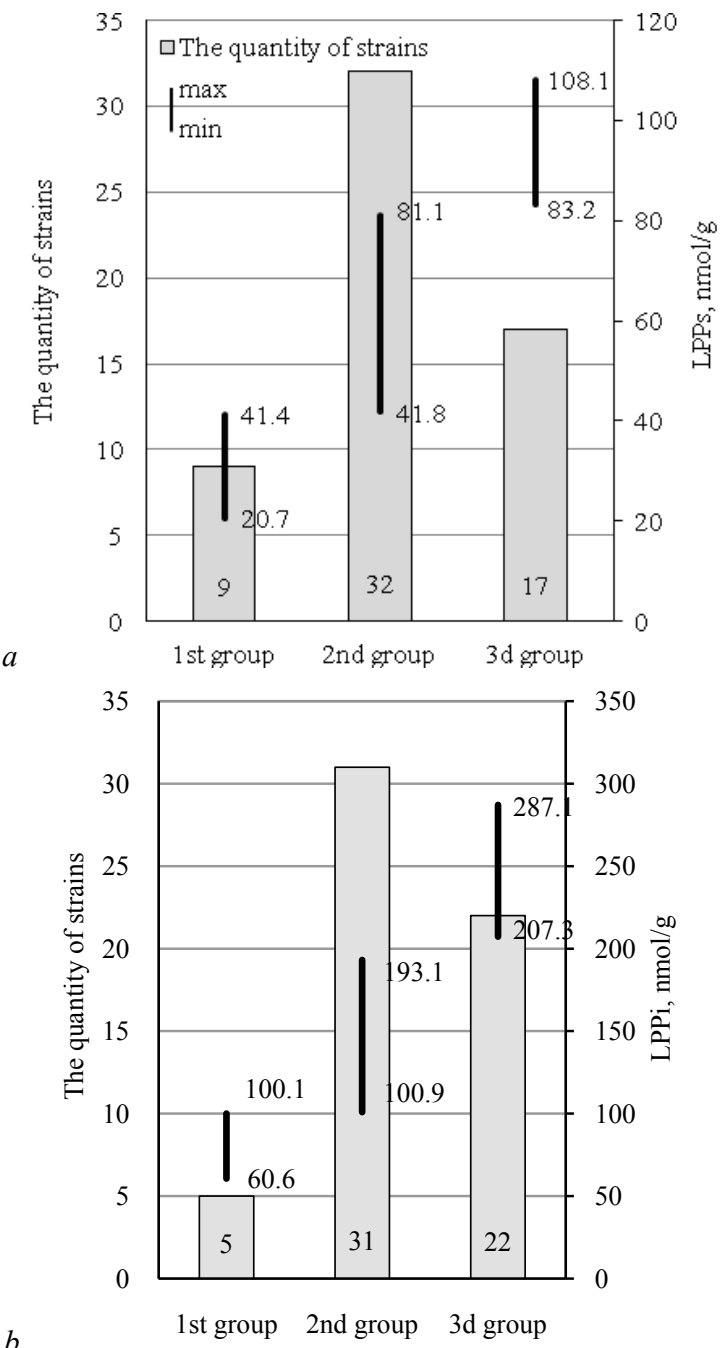

Fig. 1. The distribution of strains of Basidiomycetes according to the level of spontaneous $(a)$ and induced $(b)$ intensity of lipid peroxidation processes of mycelium on the 12th day of cultivation using periodic surface method on glucose-peptone medium (GPM)

The content of LPP products in the culture fluid of the strains was significantly lower than that of mycelium and tended to increase on the 12th day of cultivation. There are several reasons for this: firstly, cells actively regulate the synthesis and release of these products to the outside, secondly, extracellular enzymes and chemically unstable ROS cause spontaneous free radical chain reactions of destruction of compounds and their further metabolism, thirdly, GPM does not contain complex and chemically stable biopolymers, the digestion of which would intensify the exogenous processes of lipid peroxidation.
The results of determining the content of LPP products in the CF allow us to identify cultures where the level of TBA-AP significantly exceeds the average values and exceeds the mark of $10 \mathrm{nmol} / \mathrm{cm}^{3}$. These are strains $A$. cylindracea 960, L. edodes 523, P. citrinopileatus P-citr, P. eryngii P-er, P. ostreatus P-081, P-082, P-087, P-kl and P-4k. The named cultures, together with strains with a high intensity of lipid peroxidation of mycelium, have prospects of using chemically stable compounds in biotechnologies of destruction.

Induction of lipid peroxidation processes reveals certain toxic properties of initiating substances or lipid peroxidation products, or shows the resistance (or reaction) of a sample to such effects. Its results substantiate the use of indicators of the prooxidant-antioxidant system, including the intensity of LP processes, in the diagnosis of certain processes in biotechnology, medicine, and ecology.

The results of the induced intensity of the lipid peroxidation (LPPi) processes of mycelium (Fig. 1) and CF strains revealed a different response of mycological material to the action of the inducer. In all variants of the experiment, activation of LP processes specific for each strain was observed. As a result, the ratio of the induced and spontaneous intensities of the LP process was individual for each strain and did not depend on its systematic position.

Thus, the experimental data on spontaneous and induced intensity of LP processes of basidium fungi strains at 9 and 12 days of age with surface periodic cultivation on GPM allow us to state that these processes have a special level and course. Groups of cultures of basidiomycetes with different levels of LPP products were revealed. The spontaneous and induced intensity of LP processes in the mycelium of all studied strains was significantly higher than this indicator in the culture fluid. The intensity of LP processes of both mycelium and CF increased with cultivation time, which can be explained by a growing shortage of certain nutrients (primarily carbon-containing) and an increase in the number and concentration of metabolic products in the medium.

The ratio of the induced and spontaneous intensities of the LP process was individual for each strain and did not depend on its systematic position. The shift in the LPPs-LPPi equilibrium relative to the unauthorized (stationary) level of lipid peroxidation is a sign of the development of a stress reaction. Moreover, LPP products can be both inducers and primary mediators of stress as a special state of the biological system. According to the results of this study, a number of certain strains with a high level and induction of lipid peroxidation can be recommended for the involvement in the biotechnology of the destruction of chemically stable compounds or an indication of certain processes and pollutions.

As noted, the activation of LP processes is a non-specific component of the body's response to any effects that are atypical in strength and duration. At the same time, the insufficiency of antioxidant defense systems increases the degree of detection of this reaction. The increased formation of free radicals in the body and the associated increase in lipid peroxidation processes (sometimes called "oxygen stress") are accompanied by a number of disturbances in the properties of biological membranes and the functioning of cells. Oxygen stress can occur at the level of cells, tissues and the body.

The fact is that LPP are especially important in the life of xylotrophs, and the decomposition of lignin and polymer derivatives is catalyzed by lignolithic enzymes and has a free radical character, the next step was to study the effect of certain chemicals on the intensity of LPP strains $P$. eryngii P-er, F. hepatica Fh- 08 and A. cylindracea 960 (Fig. 2).

In summarizing this part of the research, we note that its results indicate an individual reaction of LPP cultures to the substances involved and the time of their exposure. The possibility of regulating lipid peroxidation processes by chemical factors of cultivation was found. The highest degree of LPP induction was recorded upon addition of: tannin by $161 \%$, after 48 hours of exposure, in the mycelium of strain Fh- 08 ; sodium lignosulfonate - by $192 \%, 48$ hours, in the mycelium of strain P-er; gallic acid - by $182 \%, 24$ hours, in the mycelium of strain P-er; hydrogen peroxide - by $257 \%, 24$ hours, in the CF of strain 960 . The biotechnological significance of this is the possibility of regulation (induction or repression) of LPP of producer strains. 

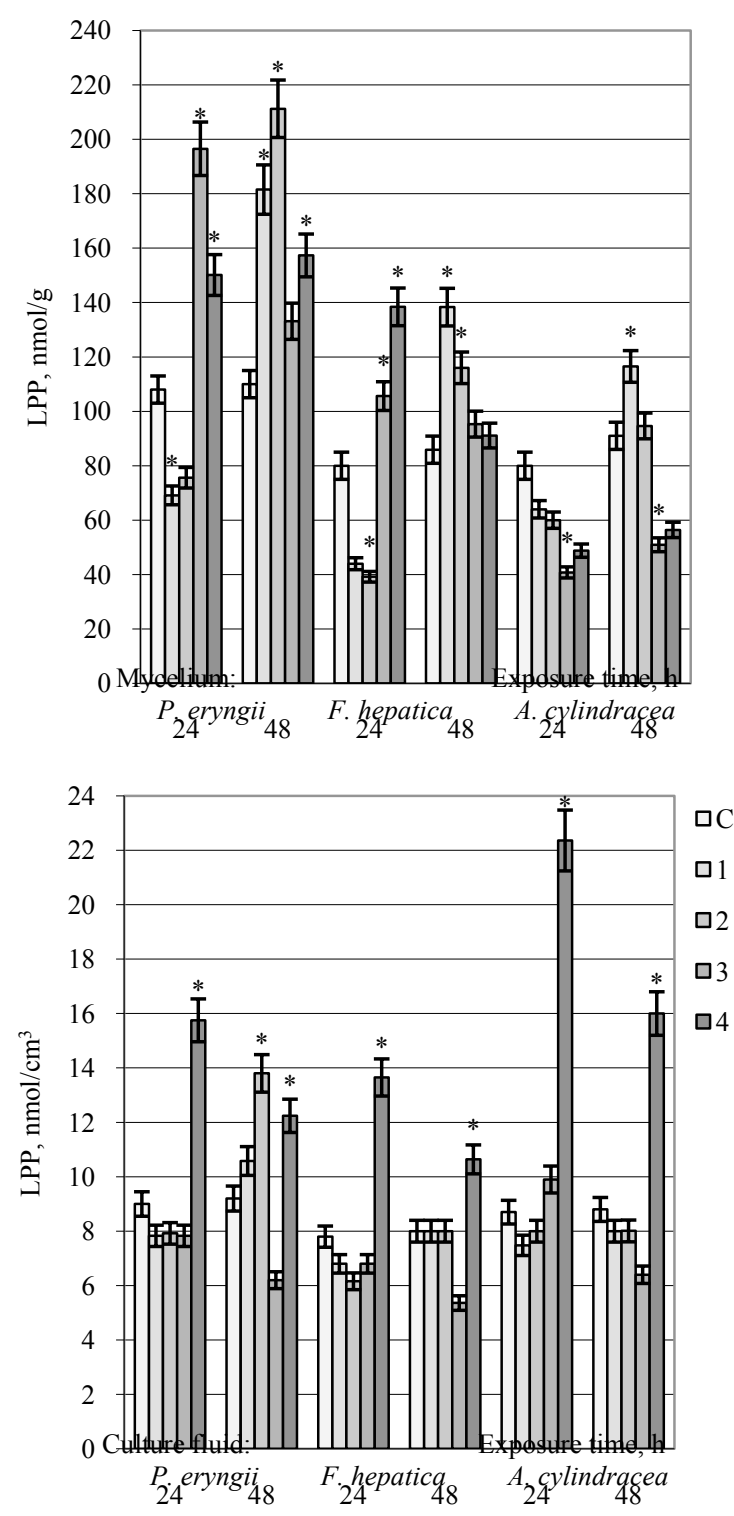

Fig. 2. The effect of tannin (1), sodium lignosulfonate (2), gallic acid (3) and hydrogen peroxide (4) on the intensity of lipid peroxidation processes of mycelium and culture fluid (CF) of strains Pleurotus eryngii P-er, Fistulina hepatica Fh-08 and Agrocybe cylindracea 960; when studying the effect, on the 10th day of culturing the strains, aqueous solutions of phenolic type substances and hydrogen peroxide up to $0.1 \%$ were aseptically added to the research flasks, and distilled water in equal volumes was added to the control flasks; the differences between the values in the control and experimental groups were determined using ANOVA, where the differences were considered significant at $\mathrm{P}<0.05$ (with Bonferroni correction); the results were defined as means \pm standard error $(\mathrm{x} \pm \mathrm{SE})$; the standard error of the mean $(\mathrm{SD})$ was calculated using analysis of variance taking into account the Bessel's correction, because a small sample was taken; ${ }^{*}$ - the difference between the experimental samples and the control (C) samples was statistically significant

\section{Discussion}

All studied strains of basidiomycetes under the applied cultivation conditions showed activity of lipid peroxidation processes, which confirms the existing scientific interest in this group - the growing number of their pharmacological, therapeutic and medical studies (Wasser, 2017; Asatiani et al., 2018; Elisashvili et al., 2018). These processes have a special level and course. Groups of cultures of basidium mu- shrooms with different levels of LPP products were revealed. The spontaneous and induced intensity of LPP in the mycelium of all studied strains is significantly higher than this indicator in the culture fluid. The intensity of the LP processes of both mycelium and CF increases with the time of cultivation, which can be explained by the growing shortage of certain nutrients (primarily carbon-containing) and an increase in the number and concentration of metabolic products in the medium (Fedotov, 2016)

An analysis of the literature on the research topic shows that numerous experiments are being conducted to study the influence of various cultivation factors, in particular chemicals, substrates, and nutrient components on the biosynthetic, including prooxidant-antioxidant activity of fungal strains (Syrchin \& Grodzinskaya, 2015; Chien et al., 2016; Elisashvili et al., 2018). However, we have not found the results of similar researches by other scientists. We suggest that the characteristic compounds that make up the lignocellulosic complex of wood or the products of its decomposition to a certain extent influence (or regulate) the prooxidant-antioxidant activity of xylotrophic basidium fungi. In particular, the choice of phenolic type chemicals and hydrogen peroxide, which affected the lipid peroxidation of the studied fungal strains, is explained as follows. The group of non-enzymatic antioxidants or precursors of their synthesis includes a number of phenolcontaining compounds. Lignosulfonates are salts of lignosulfonic acids, wastes from technological processing of plant wood raw materials, which determines their cheapness. They are natural polymers, which include substances of the aldehyde and phenolic type. They have high surface activity, and in small concentrations have a noticeable biostimulating effect. Tannin is a pharmacopeia drug made from plants and contains a group of phenolic compounds of plant origin with a large number of -OH groups. Tannins in solution form strong bonds with proteins, polysaccharides and other biopolymers. Gallic acid is a phenolic acid found in plant materials. Gallic acid esters are part of tannins and tannins and are antioxidants (Eriksson et al., 1990; Elisashvili et al., 2018). Hydrogen peroxide is the most stable of the intermediate metabolic products of hydrogen reduction. It is formed in the cells of all aerobic and facultative anaerobes growing under aerobic conditions. Hydrogen peroxide refers to reactive forms of oxygen and, with increased formation in the cell, causes oxidative stress. This substance is neutralized with the participation of antioxidative enzymes in the cytoplasm of cells and some organelles (Asatiani et al., 2011; Lushchak et al., 2016).

It was established that the used phenolic-type chemical compounds that are part of the lignocellulose complex of wood or are products of its decomposition to a certain extent affect the lipid peroxidation processes of mycelial cell lipids in the studied cultures of basidium fungi. The individual reaction of LPO processes of cultures to the applied substance and the time of its exposure were determined. The highest degree of LPO induction was recorded upon addition of: tannin - by $161 \%$, after 48 hours of exposure, in the mycelium of strain Fh-08; sodium lignosulfonate - by $192 \%, 48$ hours, in the mycelium of strain P-er; gallic acid by $182 \%, 24$ hours, in the mycelium of strain P-er; hydrogen peroxide by $257 \%, 24$ hours, in the CF of strain 960 . The biotechnological significance of this is the possibility of regulation (induction or repression) of LPO of producer strains. From a biomedical point of view, a change in the activity of the processes of lipid peroxidation of mycelium and culture fluid allows one to obtain and use more effective functional products of fungal origin, opens new directions of use and use of medicinal mushrooms.

\section{Conclusions}

Consequently, the determination and comparison of spontaneous and induced intensity of LPP allowed us to identify a group of 17 cultures of basidiomycetes that are characterized by a high content of TBA$\mathrm{AP}$ - more than $80 \mathrm{nmol} / \mathrm{h}$. These are strains of different systematic affiliation D. quercina Dq-08, F. fomentarius T-10, A. cylindracea 960, L. edodes 523, F. hepatica Fh-08, F. velutipes $\mathrm{F}-202$, P. citrinopileatus P-citr, P. eryngii P-er, P. ostreatus P-035, P-089, P-191, P-kl, P-14, P94, P-447 and P-2175, S. commune Sc-10. On the basis of high intensity 
of LP processes recorded, these cultures have prospects for the use of chemically stable compounds in the destruction of biotechnologies. The possibility of regulation (induction or repression) of LPP xylotrophs was found and a certain concentration of sodium lignosulfonate, tannin, gallic acid and hydrogen peroxide in the nutrient medium was established, which significantly affects this indicator of mycological material. The highest degree of LPP induction was recorded upon addition of: tannin - by $161 \%$, after 48 hours of exposure, in the mycelium of strain F. hepatica Fh-08; sodium lignosulfonate - by $192 \%$, 48 hours, in the mycelium of strain P. eryngii P-er; gallic acid - by $182 \%$, 24 hours, in the mycelium of strain $P$. eryngii P-er; hydrogen peroxideby $257 \%, 24$ hours, in the CF of strain A. cylindracea 960 . The obtained results require further in-depth study of the influence of phenolic-type chemicals and hydrogen peroxide on the intensity of LPO processes of basidiomycetes, however, they already indicate the possibility of regulating the processes of their biotechnological cultivation and obtaining the target product.

We express our sincere gratitude to the research associates of the Mycology Department of the M. G. Kholodny of Institute of Botany National Academy of Sciences of Ukraine for cooperation, provided materials of the Collection of Hat Mushroom Cultures (IBK), which has the status of the National Heritage of Ukraine.

\section{References}

Asatiani, M. D., Sharvit, L., Barseghyan, G. S., Chan, J. S. L., Elisashvili, V., \& Wasser, S. P. (2018). Cytotoxic activity of medicinal mushroom extracts on human cancer cells. SF Journal of Biotechnology and Biomedical Engineering, 1(1), 1-7.

Asatiani, M. D., Wasser, S. P., Nevo, E., Ruimi, N., Mahajna, J., \& Reznick, A. Z. (2011). The Shaggy Inc cap medicinal mushroom, Coprinus comatus (O.F.Mull.: Fr.) Pers. (Agaricomycetideae) substances interfere with $\mathrm{H}_{2} \mathrm{O}_{2}$ induction of the NF-kappaB pathway through inhibition of Ikappa-Balpha phosphorylation in MCF7 breast cancer cells. International Journal of Medicinal Mushrooms, 13, 19-25.

Bennett, C. F., Kwon, J. J., Chen, C., Russell, J., Acosta K., Burnaevskiy, N., Crane, M. M., Bitto, A., Vander Wende, H., Simko, M., Pineda, V., Rossner, R., Wasko, B. M., Choi, H., Chen, S., Park, S., Jafari, G., Sands, B., Perez Olsen, C., Mendenhall, A. R., Morgan, P. G., \& Kaeberlein, M. (2017). Transaldolase inhibition impairs mitochondrial respiration and induces a starvation-like longevity response in Caenorhabditis elegans. PLoS Genetics, 13(3), 10-17.

Bisko, N. A., Lomberg, M. L., Mytropolska, N. Y., \& Mykchaylova, O. B. (2016). The IBK Mushroom Culture Collection. Kyiv, M. G. Kholodny Institute of Botany, National Academy of Sciences of the Ukraine. Alterpres, Kyiv.

Chien, R. C., Lin, L. M., Chang, Y. H., Lin, Y. C., Wu, P. H., Asatiani, M. D., Wasser, S. G., Krakhmalnyi, M., Agbarya, A., Wasser, S. P., \& Mau, J. L. (2016). Anti-inflammation properties of fruiting bodies and submerged cultured mycelia of culinary-medicinal higher Basidiomycetes mushrooms. International Journal of Medicinal Mushrooms, 18(11), 999-1009.
Elisashvili, V., Kachlishvili, E., \& Asatiani, M. D. (2018). Efficient production of lignin-modifying enzymes and phenolics removal in submerged fermentation of olive mill by-products by white-rot Basidiomycetes. International Biodeterioration and Biodegradation, 134, 39-47.

Eriksson, K. E. L., Blanchette, R. A., \& Ander, P. (1990). Microbial and enzymatic degradation of wood and wood components. Springer-Verlag, Berlin.

Fedotov, O. V. (2016). The lipid peroxidation intensity of fungi strains from the orders Agaricales and Polyporales. Visnyk of Dnipropetrovsk University, Biology, Ecology, 24(2), 317-323.

Fedotov, O. V. (2016). Total antioxidant activity of some Basidiomycetes strains in growth dynamic. Biological Bulletin of Bogdan Chmelnitskiy Melitopol State Pedagogical University, 6(2), 158-165.

Fedotov, O. V., \& Bisko, N. A. (2018). Effect of phenolic substances and hydrogen peroxide on antioxidant activity of some strains of Basidiomycetes. Innovative Biosystems and Bioengineering, 2(1), 4-10.

Han, H.-S., Jacobson, A., Bilek, E. M., \& Sessions, J. (2018). Waste to wisdom: Utilizing forest residues for the production of bioenergy and biobased products. Applied Engineering in Agriculture, 34(1), 5-10.

Kapich, A. N. (2010). Oxidizability of unsaturated fatty acids and of a non-phenolic lignin structure in the manganese peroxidase-dependent lipid peroxidation system. Enzyme and Microbial Technology, 46(2), 136-140.

Kirk, P. M., Cannon, P. F., Minter, D. W., \& Stalpers, J. A. (2008). Dictionary of the fungi. $\mathrm{CABI}$, Wallingford.

Lushchak, V. I. (2011). Adaptive response to oxidative stress: Bacteria, fungi, plants and animals. Toxicology and Pharmacology, 153(2), 175-190.

Lushchak, V. I. (2014). Free radicals, reactive oxygen species, oxidative stresses and their classifications. Chemico-Biological Interactions, 224, 164-175.

Lushchak, V. I. (2016). Time-course and intensity-based classifications of oxidative stresses and their potential application in biomedical, comparative and environmental research. Redox Report, 21(6), 262-270.

Metri, Y., Warly, L., \& Suyitman. (2018). Biodegradation of lignin by white rot fungi (Pleurotus ostreatus) to Decrease the Fibre Components in the Palm Midrib. Pakistan Journal of Nutrition, 17(2), 71-75.

Obrador, E., Liu-Smith, F., Dellinger, R. W., Salvador, R., Meyskens, F. L., \& Estrela, J. M. (2018). Oxidative stress and antioxidants in the pathophysiology of malignant melanoma. Biological Chemistry, 24, 400(5), 589-612.

Syrchin, S. A., \& Grodzinskaya, A. A. (2015). Evaluation of antioxidant activity of some wild macromycetes. Ukrainian Botanical Journal, 72(3), 257-260 (in Ukrainian).

Velygodska, A. K., \& Fedotov, O. V. (2016). The production and analysis of carotenoid preparations from some strains of xylotrophic Basidiomycetes. Visnyk of Dnipropetrovsk University, Biology, Ecology, 24(2), 290-294.

Vitak, T. Y., Wasser, S. P., Nevo, E., \& Sybirna N. O. (2017). Enzymatic system of antioxidant protection of erythrocytes in diabetic rats treated with medicinal mushrooms Agaricus brasiliensis and Ganoderma lucidum (Agaricomycetes). International Journal of Medicinal Mushrooms, 19, 697-708.

Voloshko, T. E., \& Fedotov, O. V. (2011). Skryninh shtamiv bazydiomitsetiv za aktyvnistyu antyoksydantnykh oksydoreduktaz [Screening of basidiomycetes strains on the antioxidant activity of oxidoreductases]. Microbiology and Biotechnology, 16, 69-81 (in Ukrainian).

Wasser, S. P. (2011). Current findings, future trends, and unsolved problems in studies of medicinal mushrooms. Applied Microbiology and Biotechnology, 89, 1323-1332.

Wasser, S. P. (2017). Medicinal mushrooms in human clinical studies. Part I. Anticancer, oncoimmunological, and immunomodulatory activities: A review. International Journal of Medicinal Mushrooms, 19(4), 279-317. 